\title{
Mortalidade por suicídio na população brasileira, 1996-2015: qual é a tendência predominante?
}

\author{
Mortality by suicide in the Brazilian population, \\ 1996-2015: what's the predominant trend?
}

\author{
Aurean D’Eça Júnior ${ }^{1 *}$ (D), Livia dos Santos Rodrigues² (D), \\ Edivaldo Pinheiro Meneses Filho ${ }^{3}$ (D), Larissa Di Leo Nogueira Costa ${ }^{3}$ (D), \\ Adriana de Sousa Rêgo ${ }^{4}$ (D), Luciana Cavalcante Costa ${ }^{5}$ (D), \\ Rosângela Fernandes Lucena Batista 6 (D)
}

\begin{abstract}
Resumo
Introdução: O suicídio é uma das três causas de morte mais frequentes no mundo e varia conforme o sexo e a idade. Objetivo: Analisar a tendência da mortalidade por suicídio nas regiões brasileiras no período de 1996 a 2015 e sua associação com o sexo e a faixa etária. Método: Estudo ecológico de série temporal, utilizando os óbitos por suicídio analisados por regiões no período de 1996 a 2015 contidos no Sistema de Informações sobre Mortalidade. Para avaliação da tendência da mortalidade por suicídio, foi considerado o modelo de regressão de Prais-Winsten. Para comparar os percentuais de mortalidade por sexo e faixa etária, foi utilizado o teste qui-quadrado. Resultados: Observou-se tendência crescente de óbitos por suicídio nas regiões Norte $(1,73 \%$, Coef $=0,007$; p-valor $<0,001)$, Nordeste $(2,30 \%$, Coef $=0,010 ; p$-valor $=0,006)$ e Sudeste $(1,41 \%$, Coef $=0,006$; p-valor $<0,001)$ e decrescente no Sul $(-0,57 \%$, Coef $=-0,002$; p-valor $=0,001)$, além de predomínio de homens no percentual de óbitos $(p<0,001)$. Conclusão: O suicídio é considerado importante problema de saúde pública, necessitando de ações para divulgação dos riscos e programas de prevenção.
\end{abstract}

Palavras-chave: suicídio; sistemas de informação; estudos de séries temporais.

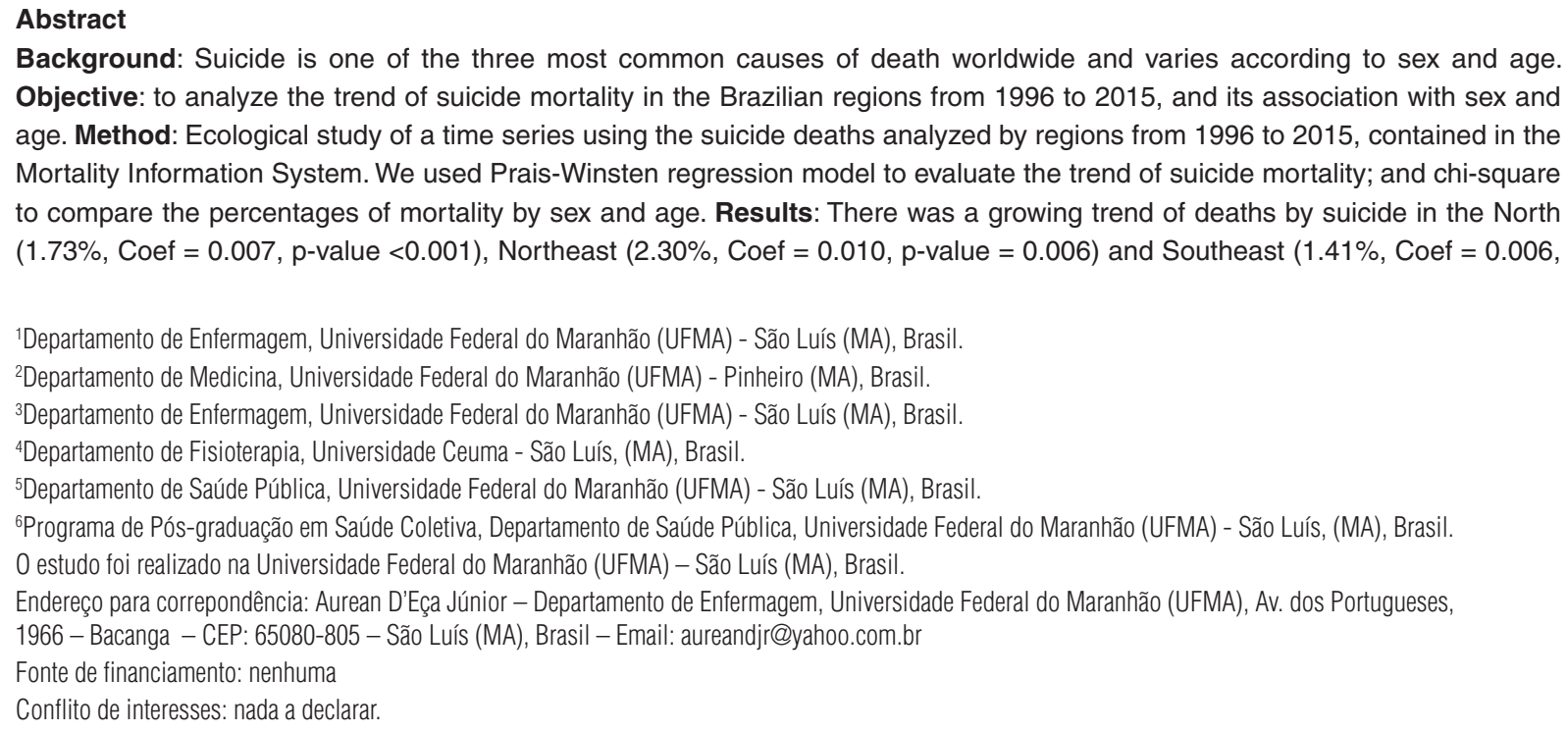

Background: Suicide is one of the three most common causes of death worldwide and varies according to sex and age. Objective: to analyze the trend of suicide mortality in the Brazilian regions from 1996 to 2015, and its association with sex and age. Method: Ecological study of a time series using the suicide deaths analyzed by regions from 1996 to 2015, contained in the Mortality Information System. We used Prais-Winsten regression model to evaluate the trend of suicide mortality; and chi-square to compare the percentages of mortality by sex and age. Results: There was a growing trend of deaths by suicide in the North $(1.73 \%$, Coef $=0.007, p$-value $<0.001)$, Northeast $(2.30 \%$, Coef $=0.010, p$-value $=0.006)$ and Southeast $(1.41 \%$, Coef $=0.006$,

'Departamento de Enfermagem, Universidade Federal do Maranhão (UFMA) - São Luís (MA), Brasil.

²Departamento de Medicina, Universidade Federal do Maranhão (UFMA) - Pinheiro (MA), Brasil.

${ }^{3}$ Departamento de Enfermagem, Universidade Federal do Maranhão (UFMA) - São Luís (MA), Brasil.

${ }^{4}$ Departamento de Fisioterapia, Universidade Ceuma - São Luís, (MA), Brasil.

${ }^{5}$ Departamento de Saúde Pública, Universidade Federal do Maranhão (UFMA) - São Luís (MA), Brasil.

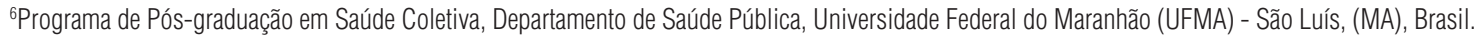

0 estudo foi realizado na Universidade Federal do Maranhão (UFMA) - São Luís (MA), Brasil.

Endereço para correpondência: Aurean D’Eça Júnior - Departamento de Enfermagem, Universidade Federal do Maranhão (UFMA), Av. dos Portugueses, 1966 - Bacanga - CEP: 65080-805 - São Luís (MA), Brasil - Email: aureandjґ@yahoo.com.br

Fonte de financiamento: nenhuma

Conflito de interesses: nada a declarar. 
$p$-value $<0.001)$ and decreasing in the South $(-0.57 \%$, Coef $=-0.002, p$-value $=0.001)$. Prevalence of men in the percentage of deaths ( $p<0.001)$. Conclusion: Suicide is considered an important public health problem, requiring actions to disseminate risk and prevention programs.

Keywords: suicide; information systems; time series studies.

\section{INTRODUÇÃO}

O suicídio é caracterizado como um ato de autolesão, ou seja, como uma ação intencional de matar a si mesmo'.

A Organização Mundial da Saúde (OMS) afirma que o suicídio se refere diretamente à agressividade, conceituando-o como um ato violento cometido contra si mesmo com a intenção de morte. Constata-se que o suicídio está entre as 20 maiores causas de morte mundiais para todas as idades e que, a cada 40 segundos, uma pessoa comete suicídio no mundo. O Brasil está entre os 10 países com os maiores números absolutos de suicídio ${ }^{2}$. Pode-se afirmar ainda que essa é uma das três causas de morte mais frequentes em muitos países do mundo e varia conforme o sexo ${ }^{3}$.

Segundo os registros da OMS, anualmente, o suicídio é responsável por 1 milhão de óbitos, o que equivale a 1,4\% do total dos óbitos ${ }^{4}$.

Acompanhando a tendência mundial, Brasil apresentou um aumento de $56 \%{ }^{5}$ nas taxas de mortalidade por suicídio entre 1980 e 2011, e o número de óbitos/100 mil habitantes subiu de 4,9 para 6,2 no período de 2000 a $2012^{6}$. Esses dados fazem com que o Brasil ocupe a oitava posição de mortalidade por suicídio entre todos os países, e essas taxas variam entre suas regiões?

Assim, o objetivo deste estudo foi analisar a tendência da mortalidade por suicídio nas regiões brasileiras no período de 1996 a 2015 e sua associação com o sexo.

\section{MÉTODO}

Trata-se de um estudo ecológico de série temporal. A população de estudo refere-se a todos os registros de óbitos por suicídio no Brasil analisados por regiões no período de 1996 a 2015. O instrumento de coleta de dados utilizado foi a declaração de óbito (DO) contida na base de dados do Sistema de Informações sobre Mortalidade (SIM), disponibilizados pelo Ministério da Saúde por meio do sítio eletrônico do Departamento de Informática do Sistema Único de Saúde (DATASUS).

Todos os óbitos autoprovocados foram considerados como suicídio, segundo a Classificação Internacional de Doenças, $10^{\mathrm{a}}$ revisão, códigos X60 a X84, contemplando análise por regiões brasileiras na série temporal de 1996 a 2015. Os coeficientes de mortalidade por suicídio foram calculados em toda a série estudada, por regiões do Brasil, correspondentes a grupos de 100 mil habitantes.

Os óbitos também foram analisados segundo o sexo (masculino e feminino) e a faixa etária (menor de 10 anos, de 10 a 19 anos, de 20 a 29 anos, de 30 a 39 anos, de 40 a 49 anos, de 50 a 59 anos e 60 anos ou mais). Para comparar e constatar diferença estatisticamente significativa $(\mathrm{p}<0,05)$ entre os percentuais de mortalidade por suicídio segundo o sexo e a faixa etária nas cinco regiões brasileiras, foi utilizado o teste qui-quadrado.

Para avaliação da tendência da mortalidade por suicídio, foi considerado o modelo de regressão de Prais-Winsten, o qual assume que os erros são correlacionados serialmente e seguem um processo autorregressivo de primeira ordem. Para ajuste do modelo, o percentual de óbitos na escala logarítmica foi considerado como a variável dependente, e o ano da série, como variável independente. Esse método vem sendo muito utilizado atualmente nos estudos epidemiológicos de análise de série temporal, uma vez que possibilita avaliar se as tendências do que se estuda serão crescentes, decrescentes ou estacionárias/estáveis, além de quantificar as taxas de variações anuais. Como é um modelo de regressão na presença de autocorrelação dos erros, sem necessidade de readequar os modelos analisados, as estimativas de tendência tendem a ser mais significativas.

Dessa forma, foi possível analisar a tendência, bem como mensurar a porcentagem de modificação de cada série temporal. A tendência foi considerada estável quando o coeficiente de regressão não diferiu de zero $(\mathrm{p}>0,05)$.

Os dados foram processados no programa Tabwin e analisados no programa STATA, versão 14.0.

As informações obtidas para a análise são de domínio público e extraídas do sítio eletrônico do DATASUS, sem a possibilidade de identificação dos indivíduos, não necessitando de aprovação prévia de comitê de ética e realizado em conformidade com as Resoluções no 466, de 12 de dezembro de 2012, e n 510, de 7 de abril de 2016, do Conselho Nacional de Saúde.

\section{RESULTADOS}

No Brasil, 172.051 óbitos por suicídio foram registrados entre 1996 a 2015. O menor número de casos ocorreu na região Norte (1.714 casos) no período de 1996-2000, enquanto o maior número ocorreu na região Sudeste (20.467 casos) no período de 2011-2015 (Tabela 1).

Aumento progressivo dos óbitos por suicídio foi observado em todas as regiões do país. A maior e a menor oscilação foram observadas, respectivamente, na região Norte, de 2,6/100 mil em 1996 para 5,0/100 mil em 2015, e na região Sul, de 7,7/100 mil em 1996 para 8,5/100 mil em 2015 (Figura 1). 
Houve diferença estatisticamente significativa entre todas as regiões brasileiras em relação ao sexo e à faixa etária, ambos com p-valor $<0,001$ (Tabela 2).

Tabela 1. Óbitos por suicídio nas regiões brasileiras no período de 1996 a 2015

Ano NORTE NORDESTE CENTRO SUDESTE SUL

$\begin{array}{llllll}1996-2000 & 1714 & 5401 & 3071 & 13570 & 10209\end{array}$

$\begin{array}{llllll}2001-2005 & 2192 & 8148 & 3802 & 15161 & 10589\end{array}$

2006-2010 $2857 \quad 10555 \quad 4011 \quad 17240 \quad 10994$

\begin{tabular}{llllll}
$2011-2015$ & 3734 & 12060 & 4585 & 20467 & 11691 \\
\hline
\end{tabular}
Analisando as tendências no período de 1996 a 2015, notou-se que elas foram crescentes nas regiões Norte, Nordeste e Sudeste, decrescente na região Sul e estável na região Centro-Oeste (Tabela 3).

A maior taxa de crescimento dos casos de suicídio ocorreu na região Nordeste, $\operatorname{com} 2,30 \%($ Coef $=0,010 ; p$-valor $=0,006)$, enquanto, nas regiões Norte e Sudeste, as taxas de crescimento foram de $1,73 \%$ (Coef $=0,007$; p-valor $<0,001)$ e 1,41\% (Coef $=0,006$; p-valor $<0,001)$, respectivamente. O decréscimo ocorrido na região Sul foi de $-0,57 \%($ Coef $=-0,002$; $\mathrm{p}$-valor $=0,001)$. A região Centro-Oeste apresentou uma tendência estacionária, com - $0,22 \%($ Coef $=-0,001 ;$-valor $=0,576)($ Tabela 3$)$.

Tabela 2. Óbitos por suicídio segundo o sexo e a faixa etária nas regiões brasileiras no período de 1996 a 2015

\begin{tabular}{|c|c|c|c|c|c|c|c|c|c|c|}
\hline \multirow[b]{2}{*}{$\operatorname{Sexo}^{*}$} & \multicolumn{2}{|c|}{ Norte } & \multicolumn{2}{|c|}{ Nordeste } & \multicolumn{2}{|c|}{ Centro-Oeste } & \multicolumn{2}{|c|}{ Sudeste } & \multicolumn{2}{|c|}{ Sul } \\
\hline & $\mathbf{N}$ & $\%$ & $\mathbf{N}$ & $\%$ & $\mathbf{N}$ & $\%$ & $\mathbf{N}$ & $\%$ & $\mathbf{N}$ & $\%$ \\
\hline Masculino & 8258 & 78,7 & 28441 & 78,7 & 12079 & 78,1 & 52266 & 78,7 & 34933 & 80,3 \\
\hline Feminino & 2235 & 21,3 & 7714 & 21,3 & 3384 & 21,9 & 14158 & 21,3 & 8550 & 19,7 \\
\hline \multicolumn{11}{|l|}{ Faixa etária* } \\
\hline$<$ de 10 anos & 21 & 0,2 & 33 & 0,1 & 7 & 0,1 & 9 & 0,2 & 15 & 0,2 \\
\hline 10 a 19 anos & 1874 & 17,9 & 3513 & 9,7 & 1843 & 11,9 & 4389 & 6,6 & 3082 & 7,1 \\
\hline 20 a 29 anos & 3421 & 32,7 & 8930 & 24,8 & 3927 & 25,5 & 15333 & 23,2 & 8229 & 19,9 \\
\hline 30 a 39 anos & 2141 & 20,5 & 7769 & 21,5 & 3350 & 21,8 & 15262 & 23,1 & 8164 & 18,8 \\
\hline 40 a 49 anos & 1386 & 13,3 & 6175 & 17,1 & 2592 & 16,8 & 13082 & 19,8 & 8670 & 18,9 \\
\hline 50 a 59 anos & 807 & 7,7 & 4320 & 12,0 & 1744 & 11,3 & 8993 & 13,4 & 6827 & 15,7 \\
\hline 60 anos ou mais & 804 & 7,7 & 5333 & 14,8 & 1938 & 12,6 & 9050 & 13,7 & 8437 & 19,4 \\
\hline
\end{tabular}

${ }^{*}$ p-valor $<0,001$

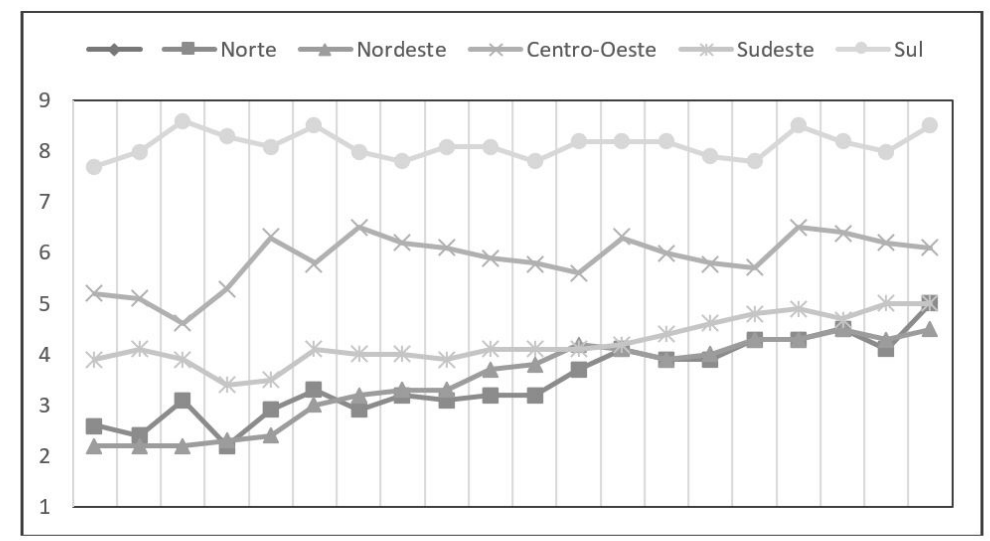

\begin{tabular}{|c|c|c|c|c|c|c|c|c|c|c|c|c|c|c|c|c|c|c|c|c|}
\hline & 1996 & 1997 & 1998 & 1999 & 2000 & 2001 & 2002 & 2003 & 2004 & 2005 & 2006 & 2007 & 2008 & 2009 & 2010 & 2011 & 2012 & 2013 & 2014 & 2015 \\
\hline$N$ & 2,6 & 2,4 & 3,1 & 2,2 & 2,9 & 3,3 & 2,9 & 3,2 & 3,1 & 3,2 & 3,2 & 3,7 & 4,1 & 3,9 & 3,9 & 4,3 & 4,3 & 4,5 & 4,1 & 5,0 \\
\hline $\mathrm{NE}$ & 2,2 & 2,2 & 2,2 & 2,3 & 2,4 & 3,0 & 3,2 & 3,3 & 3,3 & 3,7 & 3,8 & 4,2 & 4,1 & 3,9 & 4,0 & 4,3 & 4,3 & 4,5 & 4,3 & 4,5 \\
\hline $\mathrm{CO}$ & 5,2 & 5,1 & 4,6 & 5,3 & 6,3 & 5,8 & 6,5 & 6,2 & 6,1 & 5,9 & 5,8 & 5,6 & 6,3 & 6,0 & 5,8 & 5,7 & 6,5 & 6,4 & 6,2 & 6,1 \\
\hline SE & 3,9 & 4,1 & 3,9 & 3,4 & 3,5 & 4,1 & 4,0 & 4,0 & 3,9 & 4,1 & 4,1 & 4,1 & 4,2 & 4,4 & 4,6 & 4,8 & 4,9 & 4,7 & 5,0 & 5,0 \\
\hline$S$ & 7,7 & 8,0 & 8,6 & 8,3 & 8,1 & 8,5 & 8,0 & 7,8 & 8,1 & 8,1 & 7,8 & 8,2 & 8,2 & 8,2 & 7,9 & 7,8 & 8,5 & 8,2 & 8,0 & 8,5 \\
\hline
\end{tabular}

Figura 1. Evolução do coeficiente* de mortalidade por suicídio nas regiões brasileiras no período 1996 a $2015 .{ }^{*}$ Coeficiente calculado por 100 mil habitantes 
Tabela 3. Tendência da mortalidade por suicídio nas regiões brasileiras no período 19962015

\begin{tabular}{ccccc} 
Tabela 3. Tendência da mortalidade por suicídio nas regiões brasileiras no período 1996 a 2015 \\
\cline { 2 - 5 } Regiões brasileiras & \multicolumn{4}{c}{ Mortalidade por suicídio } \\
\cline { 2 - 5 } & Coeficiente & p-valor & Tendência $^{* *}$ & Taxa de variação (\%) $^{*}$ \\
Norte & 0,007 & $<0,001$ & Crescente & 1,73 \\
Nordeste & 0,010 & 0,006 & Crescente & 2,30 \\
Centro-Oeste & $-0,001$ & 0,576 & Estável & - \\
Sudeste & 0,006 & $<0,001$ & Crescente & 1,41 \\
Sul & $-0,002$ & 0,001 & Decrescente & $-0,57$ \\
\hline
\end{tabular}

${ }^{*}$ significativamente diferente de zero $(\mathrm{p}<0,05)$. ${ }^{*}$ As tendências foram classificadas como crescentes $(\mathrm{p} \leq 0,05$ e coeficiente de regressão positivo), decrescentes ( $\mathrm{p} \leq 0,05$ e coeficiente de regressão negativo) e estáveis $(\mathrm{p}>0,05)$

\section{DISCUSSÃO}

Houve tendência crescente de suicídio nas regiões Norte, Nordeste e Sudeste, decrescente na região Sul e estável na região Centro-Oeste, com predomínio significativo do sexo masculino e da faixa etária entre 20-29 anos durante o período estudado em todas as regiões.

Analisando os óbitos por suicídio, houve tendências diferentes entre as regiões e divergentes às de alguns estudos encontrados na literatura. Uma hipótese que poderia explicar esse fato seria o não registro e/ou a falta de notificação dos casos estimados em $15,6 \%$ e $13,7 \%$, respectivamente, assim como, por se tratar do preenchimento da declaração de óbito, é comum apresentar a natureza da lesão em vez da circunstância que provocou o óbito ${ }^{8}$. Há também alguns suicídios que recebem outras denominações, como afogamento e acidente automobilístico, ou são classificados como morte de causa desconhecida ou indeterminada 9 .

Um estudo realizado por Machado e Santos ${ }^{6}$ entre os anos de 2000 a 2012, utilizando o Sistema de Informação sobre Mortalidade (SIM), mostrou maiores taxas de mortalidade por suicídio nas regiões Sul (9,8 óbitos por 100 mil habitantes) e Centro-Oeste (7,6 óbitos por 100 mil habitantes), seguidas pelo Sudeste (5,6 óbitos por 100 mil habitantes), Norte (5,3 óbitos por 100 mil habitantes) e Nordeste (5,2 óbitos por 100 mil habitantes), refletindo as dimensões continentais e as diversidades culturais nacionais. No entanto, apesar da predominância do suicídio no Sul e no Centro-Oeste, a mortalidade por essa causa foi reduzida nessas regiões, enquanto houve aumento das taxas nas demais, segundo os autores citados.

Em 2009, foi criado um programa de prevenção ao suicídio (PPS) no Sul do Brasil com a organização da rede de atenção, o que culminou no fortalecimento da atenção básica, na reorganização da rede intersetorial de atendimento e no estabelecimento de uma linha de cuidados, além da capacitação profissional na identificação de riscos. Aliada ao compromisso da gestão, ao apoio político-institucional, ao controle social e à educação permanente, essa região brasileira atingiu taxas cada vez menores de casos de suicídio ${ }^{10}$.

Melhoria no preenchimento dos atestados de óbito, ações de sensibilização e notificação compulsória das tentativas também podem justificar a redução/estabilização da ocorrência dos casos de suicídio nas regiões Sul e Centro-Oeste brasileiras.

Lovisi et al. ${ }^{11}$, ao realizarem estudo descritivo das taxas de mortalidade por suicídio em regiões brasileiras e capitais no período de 1980-2006, encontraram que as capitais com taxas médias mais altas durante todo o período de estudo foram Boa Vista (7,6 mortes por 100 mil habitantes), Porto Alegre (7,3 mortes por 100 mil habitantes) e Florianópolis (6,5 por 100 mil habitantes), enquanto Salvador (1,2 morte por 100 mil habitantes) e Rio de Janeiro (2,4 mortes por 100 mil habitantes) foram as capitais com as taxas médias mais baixas. As capitais que mostraram maior crescimento das taxas de suicídio foram Goiânia (156\%), Aracaju (143\%) e Macapá (142\%).

O presente estudo encontrou percentual maior de mortes por suicídio na população masculina. Nock et al. ${ }^{12}$ também demonstraram a predominância do sexo masculino no suicídio, variando de 3 a 7,5 entre os sexos no mundo, assim como Marín-León, Oliveira, Botega ${ }^{13}$ encontraram o coeficiente médio de mortalidade por suicídio de 5,7\% no Brasil entre 2004-2010, sendo $7,3 \%$ no sexo masculino e $1,9 \%$ no feminino.

Embora as mulheres sejam propensas a tentar o suicídio mais vezes, os homens têm êxito mais frequente, o que demonstra a expressividade da ocorrência do suicídio em homens no Brasil, confirmando a tendência mundial de que eles são três vezes mais propensos do que as mulheres a cometer suicídio ${ }^{6,14}$.

É possível também que a maior ocorrência de suicídio entre os homens possa ser atribuída ao desempenho da masculinidade, o que envolve comportamentos que predispõem ao suicídio, incluindo a competitividade, a impulsividade e o maior acesso às tecnologias letais e às armas de fogo. A falência em cumprir os tradicionais papéis de gênero, que para o homem significa se constituir como provedor econômico da família, é fator de estresse. Inseridos em uma cultura patriarcal, os homens são mais sensíveis a reveses econômicos, como desemprego e empobrecimento, e mais propensos ao suicídio ${ }^{15,16}$, enquanto as mulheres apresentam mais fatores protetores, como a prática religiosa, o reconhecimento precoce dos fatores de risco e a busca por ajuda e/ou suporte mental ${ }^{17,18}$.

No Brasil, segundo a Portaria $n^{\circ} 1.876$ de $2006^{19}$, as mortes por suicídio podem ser evitadas por meio de ações de promoção 
e prevenção em todos os níveis de atenção à saúde de forma articulada entre o Ministério da Saúde, as Secretarias de Estado de Saúde, as Secretarias Municipais de Saúde, as instituições acadêmicas, as organizações da sociedade civil, os organismos governamentais e os não governamentais, nacionais e internacionais.

Mesmo que, em algumas regiões brasileiras, a notificação dos casos de suicídio seja um compromisso da gestão pública, são consideradas limitações aos estudos sobre suicídio que merecem ser enfatizadas: consideráveis subnotificações dos dados relacionadas ao estigma social, razões jurídicas e questões religiosas. Ainda, a transcrição de outras denominações para a causa da morte, por exemplo, afogamento, envenenamento acidental, acidente automobilístico, e a morte por causa indefinida dificultam a abrangência real do problema.

Mediante o exposto, o suicídio é considerado um importante problema de saúde pública no Brasil, sendo imperativo o desenvolvimento de ações educativas que visem à divulgação dos riscos, programas de prevenção e complicações relacionadas ao suicídio, priorizando a atenção primária e tendo como alvo tanto a população geral quanto os grupos de risco específicos, visando, assim, melhorar as estatísticas apresentadas.

O presente trabalho evoca a necessidade de articulação intersetorial entre as esferas da gestão federal, estadual e municipal que contemple ações educativas de promoção e de prevenção ao suicídio. Os dados epidemiológicos despertam para a troca de experiências que permitirão tratar o suicídio como um problema de saúde pública em nível nacional e que carece do apoio político, dos profissionais de saúde, dos profissionais da segurança pública e da sociedade como um todo. A pesquisa também sugere a realização de mais estudos para desfazer a visão preconceituosa que se tem do suicídio e estimular, por parte da gestão, programas que elevem o problema como uma questão social que necessita de soluções coletivas envolvendo a comunidade.

\section{REFERÊNCIAS}

1. Gomes JO, Baptista MN, Carneiro AM, Cardoso HF. Suicídio e internet: análise de resultados em ferramentas de busca. Psicol Soc. 2014;26(1):6373. http://dx.doi.org/10.1590/S0102-71822014000100008.

2. Alves MA, Cadete MM. Tentativa de suicídio infanto-juvenil: lesão da parte ou do todo? Cien Saude Colet. 2015;20(1):75-84. PMid:25650600.

3. Freitas JL, Prado AS, Mathias B, Greschuck GR, Dequech J No. Revisão bibliométrica das produções acadêmicas sobre suicídio entre 2002 e 2011. Psicol Pesqui. 2013;7(2):251-60. http://dx.doi.org/10.5327/Z19821247201300020013.

4. World Health Organization. Country reports and charts available [Internet]. Geneva: WHO; 2014 [citado em 2018 Mai 21]. Disponível em: www.who. int/mental_health/prevention/sui cid e/country_rep orts/en/index

5. Waiselfisz JJ. Mapa da violência: homicídios e juventude no Brasil Atualização 15 a 29 anos. Brasília: Secretaria-Geral da Presidência da República, Secretaria Nacional de Juventude, Secretaria de Políticas de Promoção da Igualdade Racial; 2014.

6. Machado DB, Santos DN. Suicídio no Brasil, de 2000 a 2012. J Bras Psiquiatr. 2015;64(1):45-54. http://dx.doi.org/10.1590/0047-2085000000056.

7. Coêlho BM, Andrade LH, Borges G, Santana GL, Viana MC, Wang YP. Do childhood adversities predict suicidality? Findings from the general population of the metropolitan area of São Paulo, Brazil. PLoS One. 2016;11(5):e0155639. http://dx.doi.org/10.1371/journal.pone.0155639. PMid:27192171.

8. Instituto Brasileiro de Geografia e Estatística. Tábuas completas de mortalidade - 2006. Rio de Janeiro: IBGE; 2013.

9. Gotsens M, Marí-Dell’Olmo M, Rodríguez-Sanz M, Martos D, Espelt A, Pérez $\mathrm{G}$, et al. Validación de la causa básica de defunción en las muertes que requieren intervención medicolegal. Rev Esp Salud Publica. 2011 abr;85(2):163-74. http://dx.doi.org/10.1590/S1135-57272011000200005. PMid:21826379.

10. Conte M, Meneghel SN, Trindade AG, Ceccon RF, Hesler LZ, Cruz CW, et al. Programa de prevenção ao suicídio: estudo de caso em um município do sul do Brasil. Cienc Saude Colet. 2012;17(8):2017-26. http://dx.doi. org/10.1590/S1413-81232012000800013.

11. Lovisi GM, Santos SA, Legay L, Abelha L, Valencia E. Análise epidemiológica do suicídio no Brasil entre 1980 e 2006. Rev Bras Psiquiatr. 2009;31(Suppl 2):86-96. http://dx.doi.org/10.1590/S1516-44462009000600007.

12. Nock MK, Borges G, Bromet EJ, Cha CB, Kessler RC, Lee S. Suicide and suicide behavior. Epidemiol Rev. 2008;30(1):133-54. http://dx.doi. org/10.1093/epirev/mxn002. PMid:18653727.

13. Marín-León L, Oliveira HBD, Botega NJ. Suicide in Brazil, 2004-2010: the importance of small counties. Rev Panam Salud Publica. 2012;32(5):351-9. http://dx.doi.org/10.1590/S1020-49892012001100005. PMid:23338692.

14. Meneghel SN, Victora CG, Faria NMX, Carvalho LAD, Falk JW. Características epidemiológicas do suicídio no Rio Grande do Sul. Rev Saude Publica. 2004;38(6):804-10. http://dx.doi.org/10.1590/S0034-89102004000600008. PMid:15608898.

15. Leal OF. Suicídio, honra e masculinidade na cultura gaúcha - Cultura e Identidade masculina. Cadernos de Antropología. 1992;6:7-21.

16. Minayo MCDS, Meneghel SN, Cavalcante FG. Suicídio de homens idosos no Brasil. Cien Saude Colet. 2012;17(10):2665-74. http://dx.doi.org/10.1590/ S1413-81232012001000016. PMid:23099754.

17. Bando DH, Brunoni AR, Fernandes TG, Benseñor IM, Lotufo PA. Suicide rates and trends in São Paulo, Brazil, according to gender, age and demographic aspects: a joinpoint regression analysis. Rev Bras Psiquiatr. 2012;34(3):28693. http://dx.doi.org/10.1016/j.rbp.2012.02.001. PMid:23429774.

18. Vidal CEL, Gomes CB, Mariano CA, Leite LMR, Silva RA, Lasmar SC. Perfil epidemiológico do suicídio na microrregião de Barbacena, Minas Gerais, no período de 1997 a 2012. Cad Saude Colet. 2014;22(2):158-164. http://dx.doi.org/10.1590/1414-462X201400020008.

19. Brasil. Ministério da Saúde. Portaria $\mathrm{n}^{\circ} 1876$, de 14 de agosto de 2006. Institui Diretrizes Nacionais para Prevenção do Suicídio, a ser implantadas em todas as unidades federadas, respeitadas as competências das três esferas de gestão. Diário Oficial da República Federativa do Brasil, Brasilia, 15 de agosto de 2006; Seção 1, p. 65.

Recebido em: Maio 22, 2018 Aprovado em: Dez. 13, 2018 